\title{
Design and Research of Intelligent Greenhouse Monitoring System Based on Internet of Things
}

\author{
Li Fu-juan \\ Collage of Mechanical Engineering, Yanshan University \\ Qinhuangdao China \\ Wang Zhi-song \\ Collage of Mechanical Engineering, Yanshan University \\ Qinhuangdao China \\ Zhao Xin-yuan \\ Collage of Mechanical Engineering, Yanshan University \\ Qinhuangdao China
}

\author{
Li Shi-hua \\ Collage of Mechanical Engineering, Yanshan University \\ Qinhuangdao China \\ shli@ysu.edu.cn \\ Chen Zhong-fei \\ Collage of Mechanical Engineering, Yanshan University \\ Qinhuangdao China
}

\begin{abstract}
Since the greenhouse has characters of temperaturehumidity uneven distribution, strong coupling and traditional control strategy can't achieve the ideal control effect, the greenhouse monitoring system based on internet of things (IOT) is designed. The system uses a fuzzy neural network which has good control effectiveness in complex and changeable greenhouse system. Perception layer using ZigBee protocol for wireless communications, and greenhouse environment can be controlled by site control software/master node, which makes the system more effective and stronger. The master node uses mobile communication network to send sensing layer data to application layer and can send alarm SMS. This system has the characteristics of low cost, simple structure, flexible networking and easy extending, which adapts to the requirements of complex greenhouse control.
\end{abstract}

Keywords-IOT; fuzzy neural network; ZigBee; remote monitoring

\section{INTRODUCTION}

The application and development in the field of internet of things in agriculture are particularly quickly in today's society. The intelligent agriculture based on internet of things will be more and more. Wireless sensor network (WSN), which is the core of the IOT technology, has the advantages of ad-hoc network, simple deployment, low energy consumption and easy manage[1-3]. Wireless sensor network is a typical multiple hops network of ad-hoc network, and its selforganizing function can guarantee the network connected in the case of damage of part of the nodes. In the greenhouse environment, because of the uneven distribution of temperature and humidity, it requires a large number of sensors to monitor environmental information, and the big inertia of greenhouse environment leads to the slow change of quantity of state information[4-6]. These features make wireless sensor network widely used in the field of greenhouse control. In the 21 st century, with the continuous development of radio technology, and the coverage of each mobile operator

Hebei province science and technology research and development plan scientific support program(14227204D) network is becoming more and broader and mobile communication technology is becoming more and more mature, intelligent greenhouse develops in the direction of wireless technology and remote monitoring.

For the greenhouse environment system is a large inertia nonlinear multivariable system, there are phenomenon such as delay and coupling, so it is difficult to set up a precise mathematical model in such a system[7-10]. Fuzzy neural network has membership function and inference rules to adjust fuzzy inference system. Combined with the advantages of both, it can well meet the demand for control of large inertia and nonlinear system. At the same time, the learning ability of neural network successfully avoids the subjectivity of fuzzy control rules and membership functions and the precision of control is improved[11-13]. By using fuzzy neural network for intelligent control inside the greenhouse environment for temperature and humidity, it can achieve very good effects.

\section{GENERAL DESIGN OF SYSTEM}

This system consists of three layer structures, which are sensing layer, network layer and application layer, just as shown in Figure 1.

The sensing layer is composed of wireless communication nodes with ad-hoc network capacity and is arranged in the greenhouse. A wireless communication node contains an acquisition node, a control node and an execution driven node. The acquisition node is used to collect information collected by all sensors in the greenhouse. The control node receives the information sent by the acquisition node (temperature, humidity, $\mathrm{CO}$ concentration, light intensity, etc.), and then gathers it to the gateway node, and sends control commands to execution driven node at the same time. The execution driven node receives the control commands and will open or close the corresponding device. The communications between each node of the sensing layer use ZigBee wireless communication technique [14-16]. 
The network layer is GPRS (2.5G) network, which has basically covered all rural areas. In addition, the data volume of greenhouse monitoring is not very large. The most important is that once the GPRS applications are activated, it will not drop unless it is shut down. The equipment will change into the resting state automatically when there is no data transferred, and also won't produce cost of traffic. These advantages well adapts to the needs of ordinary farmers.

The master node will send data collected by the acquisition node to the application software through the GPRS module and will send alarm messages to the user's mobile phone when the data of the sensors is abnormal. At the same time, the user can use the application layer software of the greenhouse for remote control and real-time monitoring.

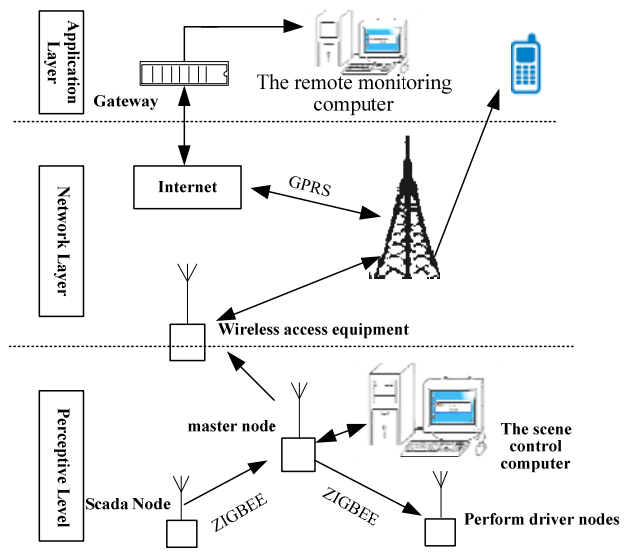

Figure 1 System structure

\section{THE DESIGN OF THE MAIN FUNCTION MODULE OF SYSTEM}

\section{A. The design of each node of perception layer}

System data is acquired and transmitted by microprocessor integrated by CC2530 ZigBee module, RS232 serial output module, power module, real-time clock module, a debugging circuit module and other components.

Because of the uneven distribution of temperature in the greenhouse, so the use of multi-point temperature measurement method is chosen for temperature acquisition. Taking into account the cost and accuracy issues, the final selection of a digital sensor is DS18B20 as a temperature sensor; a single-bus multi-point network for temperature acquisition. The Single-bus multi-point temperature measurement circuit is shown in Figure 2.

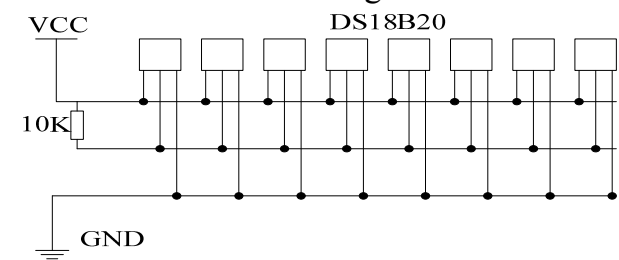

Figure $\overline{\overline{2}}$ 1-wire circuit of multi-point temperature measurement

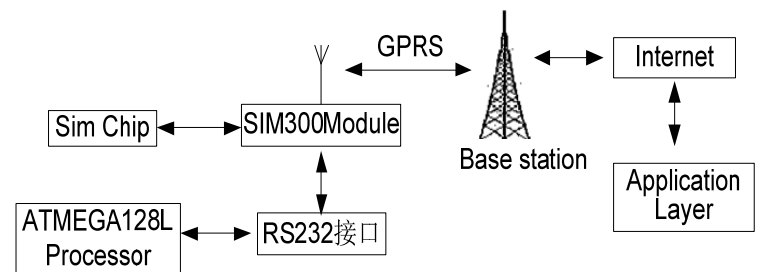

Figure 3 Hardware architecture of communication module SIM300

B. The equipment of wireless access

According to the site situation, GPRS is chosen as mobile communication network access to the internet. The SIM300 module chip is selected as a wireless access device. The hardware of SIM300 mainly includes the SIM card module circuit, indicator light, the master node processor Atmega128L and the serial port RS232. The communication module principle of hardware structure is shown in figure 3 .

\section{C.The design of the fuzzy neural network controller}

Greenhouse system is a coupling complex system, which is relatively complicated controlled. To this end, the system establishes a dual-input temperature and humidity fuzzy neural network control model. According to actual needs, implemented agencies are chosen, namely: wet curtain fan Y1, shade net Y2, circulating fan Y3, sunroof (side windows) Y4, heater Y5, micro-mist humidifier Y6.

Enter the amount of error of temperature and humidity, the basic domain X1 of temperature error is $[6,6]$, setting the temperature control system domain error fuzzy on $\{-6,-5,-4$, $3,-2,-1,0,1,2,3,4,5,6\}$, the amount of blur temperature error is divided into seven levels: NB, NM, NS, ZO, PS, PM, PB. Basic domain $\mathrm{X} 2$ of humidity error is $[-4,4]$. Fuzzy set theory of humidity error is $\{-4,-3,-2,-1,0,1,2,3,4\}$, and the amount of humidity error blurring is divided into five levels: NB, NS, O, PS, PB. The membership function of this system [17] using trigonometric functions, temperature and humidity membership function of the image will be shown in Figure 4.
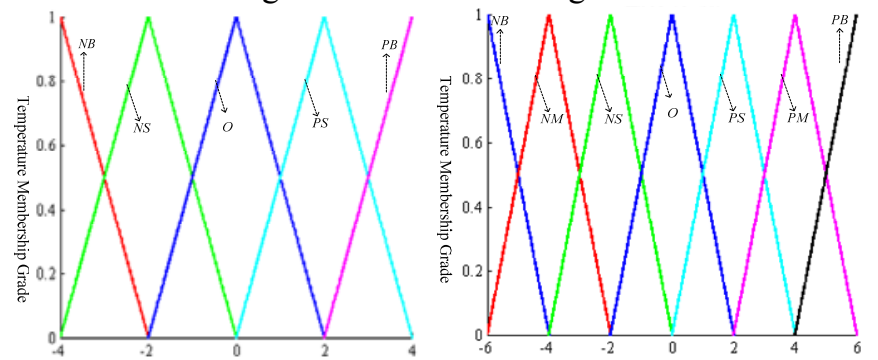

Figure 4 Temperature-Humidity membership function image

In the output, Sun-shade net、Circulation fan、Skylight (side window)、Micro mist humidifier are with just two states, from 0 to be shut down, from 1 to be open. Wet curtain wind and heater are multi-stage control units, Wet curtain air set can be divided into four levels: open a few fan, open the most fan, open all. So its corresponding fuzzy language is Z0, PS,

PM, PB. The heater is divided into four levels: Closing, short heating time, the appropriate time heating, heating for a long time. Its corresponding fuzzy language is O, PS, PM, PB. Because of the temperature error is divided into seven levels, humidity error is divided into five grades, So the control rules for 35 , Control table as shown in table 1 . 
Table 1 Fuzzy-control rule table

\begin{tabular}{cccccccc}
\hline $\boldsymbol{X} \mathbf{1}$ & $\boldsymbol{X} \mathbf{2}$ & $\begin{array}{c}\text { The } \\
\text { heater }\end{array}$ & $\begin{array}{c}\text { Wet } \\
\text { curtain } \\
\text { fan }\end{array}$ & $\begin{array}{c}\text { Circu- } \\
\text { lation } \\
\text { fan }\end{array}$ & $\begin{array}{c}\text { Sun- } \\
\text { shade } \\
\text { net }\end{array}$ & $\begin{array}{c}\text { Side } \\
\text { window } \\
\text { skylight }\end{array}$ & $\begin{array}{c}\text { Micro } \\
\text { mist } \\
\text { humid- } \\
\text { ifier }\end{array}$ \\
\hline NB & NB & O & NB & 1 & 1 & 1 & 0 \\
& NS & O & NB & 1 & 1 & 1 & 0 \\
& O & O & NB & 0 & 1 & 0 & 0 \\
& PS & O & NB & 0 & 1 & 0 & 0 \\
NM & PB & O & NB & 0 & 1 & 0 & 0 \\
& NB & O & NM & 1 & 1 & 1 & 0 \\
& NS & O & NM & 1 & 1 & 1 & 0 \\
\hline
\end{tabular}

All kinds of possible situations in the process of control and the corresponding control strategies are classified and summarized. "IF-THEN" form of conditional statements is used to describe the control rules. Part of the conditional statements are as follows:

If $\mathrm{E} 1=\mathrm{NB}$ and $\mathrm{E} 2=\mathrm{NB}$ then $\mathrm{Y} 1=\mathrm{NB}$ and $\mathrm{Y} 2=\mathrm{BB}$ and $\mathrm{Y} 3=1$ and $\mathrm{Y} 4=1$ and $\mathrm{Y} 5=\mathrm{O}$ and $\mathrm{Y} 6=0$

......

In establishing fuzzy control rules, the principle of the selection of control quantity is: the temperature control is the main contradiction, and when conflict occurs between the temperature and humidity control, temperature control is preferred; when the error is big or large, select the control to eliminate the error as soon as possible.

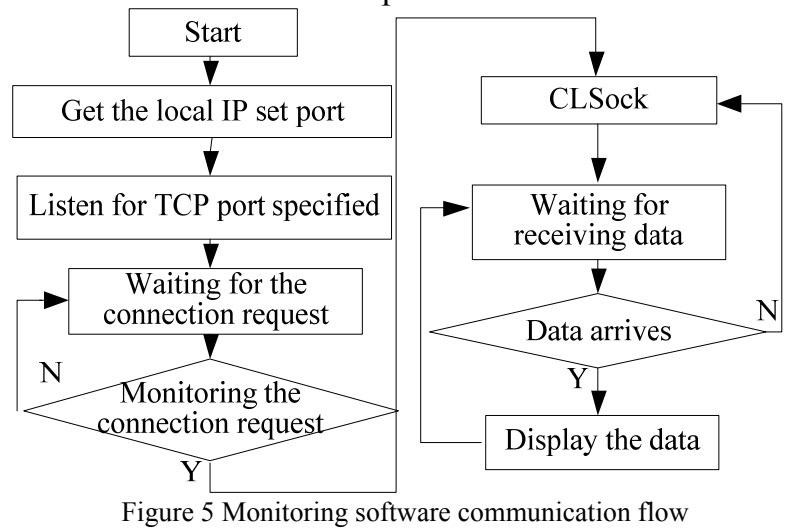

IV. APPLICATION EXPERIMENTS

The test is conducted in a greenhouse of Qinhuangdao. To verify the stability of this system, carry out the experiment of data interrupt and short-message warning. Cutting down the power, sampling nodes stop sending data and master node sounds a warning. The power recovers, this system restarts to send data, and the warning disappear, and there is no disorder, crash or other undesirable phenomenon. Heating the air around temperature sensors, to make temperature higher than warning value, product testing institute receives alarm message in time, no significant delay. As shown in figure 6.

Practical experiment includes automatic control and manual control. When there is no one controlling the system, the master node automatically controls the executor node through wireless network. If someone controls the system on the computer, the local control software sends control command to master node by serial communication, then master node carries out UART interrupt and controls the executor node. This experiment is designed to test real-time performance of master node and serial communication data veracity of local control software. The status of local control software is shown in figure 7.
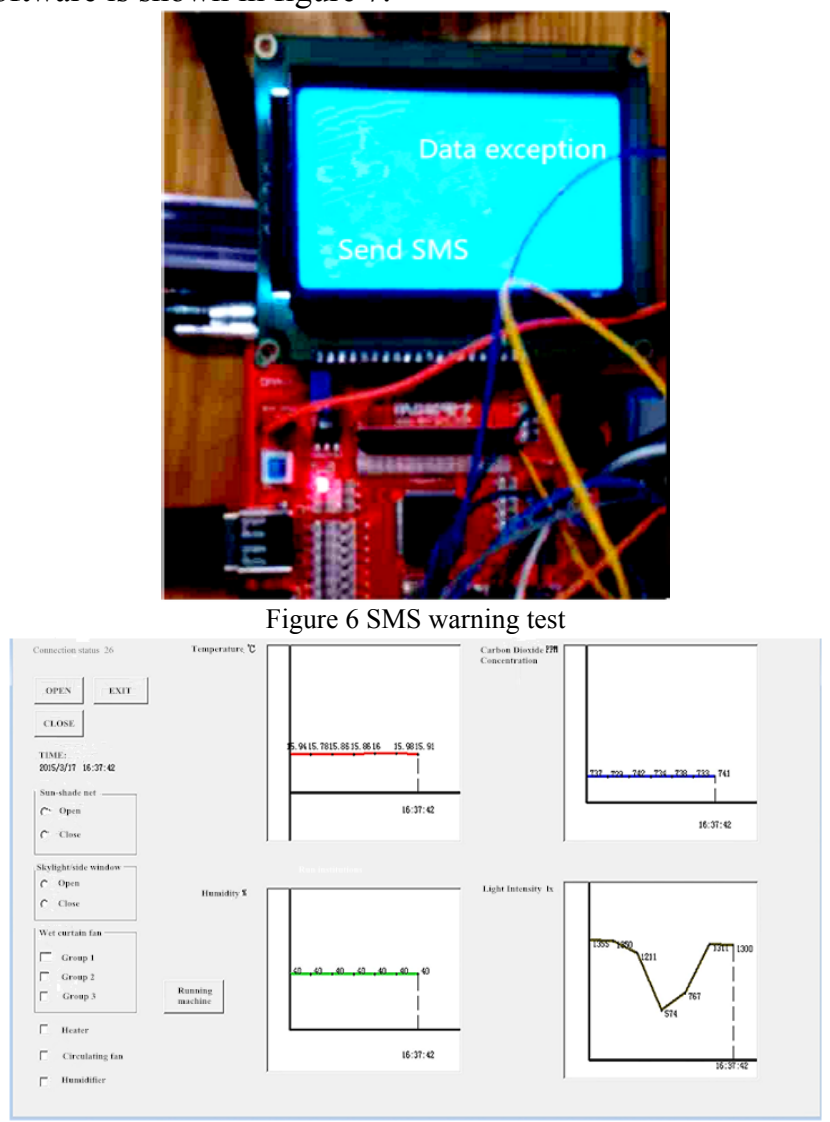

Figure 7 Living control test

The field experiment proves that the system responds well. Accuracy of data acquisition can meet the demand of control and feedback data in time and exactly. It can thus be seen that the sensing layer communications, master node command execution and communication between control software and master node run well.

\section{CONCLUSIONS}

This paper take a full consideration of cost, practicability and other factors, combining the IOT with fuzzy neural network control method, using GPRS to remote control, designing an intelligent greenhouse monitoring system with stable performance, simple structure and easy extensibility. The advantages are as follows:

1) Based on fuzzy neural network and wireless sensor network, resolves the greenhouse problems like great inertia, coupling and difficulty to control in some way.

2) Using wireless network, resolves the problems like large number of wiring, high cost and so on.

3 ) Compared to traditional greenhouse, the intelligent greenhouse based on IOT has the characteristics of low cast, 
higher automation, and reasonable layout. It can meet the needs of further development of intelligent agriculture.

\section{References}

[1] ITU Strategy and Policy Unit (SPU) .ITU internet reports 2005: The internet of things[C]. Geneva: International Telecommunication Union (ITU), 2005.

[2] Li Daoliang. Internet of things and wisdom agriculture[J]. Agricultural Engineering, 2012(1): 003.

[3] Hu Jin, Fan Hongpan, Zhang Haihui, Zhou Qingzhen, Liang Yan, Liu Zhengdao, Liu Xiang. Design of regulation system of light environment in greenhouse based on wireless sensor network [J]. Transactions of the Chinese Society of Agricultural Engineering, 2014, 30(4): 160-167.

[4] Lin Hong. Research on intelligent greenhouse environmental monitoring system [J]. Journal of Agriculture University of Hebei, 2014(05): 130134.

[5] Ole Green, Esmaeil S. Nadimi, Victoria Blanes-Vidal, Rasmus N. Jørgensen, Ida M.L. Drejer Storm, Claus G. Sørensenl. Monitoring and modeling temperature variations inside silage stacks using novel wireless sensor networks $[\mathrm{J}]$. Computers and Electronics in Agriculture, 2009, 69(2):149-157.

[6] Wang Fulu, Fang Junlong, Zhang Xihai. The Environmental Monitoring System for Greenhouse Based on Wireless Sensor Network[J]. TECHNIQUES OF AUTOMATION AND APPLICATIONS. 2009(10): 61-63.

[7] Guo Wenchuan, Cheng Hanjie, Li Ruiming. Greenhouse Monitoring System Based on Wireless Sensor Networks [J]. Transactions of the Chinese Society for Agricultural Machinery, 2010, 41(7): 181-185.
[8] Wang Dandan, Zong Zhenhai, Chen Huishan, Jian Xingliang. Remote intelligent greenhouse control design based on STM32 [J]. Acta Agriculturae Zhejiangensis, 2014, 26(3): 791-796.

[9] Han Huafeng, Du Kerning, Sun Zhongfu, Zhao Wei, Chen Ran, Liang Jvbao. Design and application of ZigBee based telemonitoring system for greenhouse environment data acquisition[J]. Transactions of the CSAE, 2009(7): 158-163.

[10] Yang Wei, Lv Ke, Zhang Dong, Wu Song, Long Zhiqiang, Shang Shouhai. Development of wireless intelligent control terminal of greenhouse based on ZigBee[J]. Transactions of the CSAE, 2010 (3): 198-202.

[11] Chen Chunling, Bai Shuang, Li Tianlai, Xv Tongyu. Research of North Solar Greenhouse Control System Base on the Fuzzy Control Algorithm[J]. Journal of Shenyang Agricultural University(Social and Edition), 2013-06, 44(3): 262-267.

[12] Li Yingxia, Du Shangfeng. Advances of intelligent control algorithm of greenhouse environment in China[J]. Transactions of the CSAE, 2004, 20: 267-272.

[13] Du Xiaokun. The Study of Fuzzy Neural Networks controller of Greenhouse System[J]. Control \& Automation, 2010, 22: 55-56.

[14] Mao Pengjun, Jiang Shui, Wang Jun, Zhang Fu ,Qiu Zhaomei. Study on segmentation and diagnosis of wine grape disease based on image processing[J]. Journal of Chinese Agricultural Mechanization, 2015 (1): 102-115.

[15] Sheng Ping, Guo Yangyang, Li Pingping. Intelligent Measurement and Control System of Facility Agriculture Based on ZigBee and 3 G[J]. Transactions of the Chinese Society for Agricultural Machinery, 2012, 43(12): 229-233.

[16] Pang Na, Cheng Fude. Design of Greenhouse Monitoring System Based on ZigBee Wireless Sensor Networks[J]. Journal of Jilin University (Information Science Edition), 2010(1): 55-60.

[17] Jiang Hui, Wang Huiqiang, Jiang Yunfei, Zhang Faming, Shi Lixi. Greenhouse environmental control system based on fuzzy control[J]. Journal of Agriculture University of Hebei, 2008(03): 108-111. 